\title{
PAMAM dendrimer roles in gene delivery methods and stem cell research
}

\begin{abstract}
Nanotechnology has provided new technological opportunities, which could help in challenges confronting stem cell research. Polyamidoamine (PAMAM) dendrimers, a new class of macromolecular polymers with high molecular uniformity, narrow molecular distribution specific size and shape and highly functionalised terminal surface have been extensively explored for biomedical application. PAMAM dendrimers are also nanospherical, hyperbranched and monodispersive molecules exhibiting exclusive properties which make them potential carriers for drug and gene delivery.
\end{abstract}

Keyword: Gene delivery; PAMAM dendrimer; Stem cell 